\title{
Análise do Programa de Controle da Tuberculose no estado do Pará, Brasil, de 2005 a 2014
}

\section{Analysis of the Tuberculosis Control Program in Pará State, Brazil, from 2005 to 2014}

Dilma Costa de Oliveira Neves', Luana de Oliveira Loureiro', Nathalya Pinheiro Paiva', Maria Deise de Oliveira Ohnishi ${ }^{1}$, Claudia Daniela Tourão Ribeiro'

' Centro Universitário do Estado do Pará, Belém, Pará, Brasil

\section{RESUMO}

OBJETIVO: Analisar indicadores do Programa Nacional de Controle da Tuberculose nas Regiões de Integração do estado do Pará, de 2005 a 2014. MATERIAIS E MÉTODOS: Estudo ecológico, utilizando 31.372 casos de tuberculose (TB) pulmonar em pessoas com idade a partir de 20 anos, registrados no Sistema de Informação de Agravos de Notificação. RESULTADOS: A incidência média no Estado foi de 71,7/10.000 hab. A Região Metropolitana registrou 49,6\% (15.572) dos casos e incidência de 1 14,7/10.000 hab., com tendência crescente no período (22,3\%). A maior redução no número de casos ocorreu na Região Xingu $(41,9 \%)$. No Pará, $70,8 \%$ dos casos receberam alta por cura, com maior proporção na Região Guamá $(77,9 \%)$. Apenas $72,6 \%$ dos casos tiveram confirmação laboratorial. A taxa de alta por cura, nos casos confirmados, reduziu de 73,1\%, em 2005, para 67,3\%, em 2014, com a maior taxa (85,4\%) em 2006, na Região Rio Caeté; e a menor taxa (48,9\%) em 2014, na Região Tapajós, com tendência de aumento nas Regiões Araguaia, Carajás, Lago de Tucuruí e Tocantins. Em cinco Regiões, o abandono de tratamento foi superior à média do Estado (13,0\%) e, no período, aumentou nas Regiões Xingu, Tapajós, Marajó, Tocantins, Carajás, Guamá, Rio Capim e Rio Caeté. A maior mortalidade ocorreu na Região Metropolitana em 2011; e a menor, na Região Guamá, nos anos de 2009 e 2010. CONCLUSÃO: No período estudado, o estado do Pará encontrava-se distante de alcançar os indicadores propostos para a eliminação da TB, contribuindo para a manutenção da endemia no Brasil.

Palavras-chave: Tuberculose; Incidência; Avaliação de Programas e Projetos de Saúde; Estudos Ecológicos.

\begin{abstract}
OBJECTIVE: To analyze the indicators of the Brazilian National Tuberculosis Control Program in the Integration Regions of Pará State, from 2005 to 2014. MATERIALS AND METHODS: Ecological study using 31,372 cases of pulmonary tuberculosis (TB) in people aged 20 years and older, registered in the Notifiable Diseases Information System. RESULTS: The average incidence in all State was 71.7/10,000 inhabitants. Região Metropolitana recorded $49.6 \%(15,572)$ of the cases and incidence of $114.7 / 10,000$ inhabitants, with increasing trend in the period (22.3\%). The greatest reduction in the number of cases occurred in Região Xingu (41.9\%). In Pará, $70.8 \%$ of cases were discharged by cure, with the highest proportion in Região Guamá (77.9\%). Only $72.6 \%$ of the cases had laboratory confirmation. The rate of discharge by cure, in confirmed cases, decreased from $73.1 \%$ in 2005 to $67.3 \%$ in 2014, with the highest rate (85.4\%) in 2006, in Região Rio Caeté; and the lowest rate (48.9\%) in 2014, in Região Tapajós, with a tendency to increase in Araguaia, Carajás, Lago de Tucuruí, and Tocantins Regions. In five Regions, the discontinuing treatment was higher than the State average (13.0\%), and, in the period, it increased in Xingu, Tapajós, Marajó, Tocantins, Carajás, Guamá, Capim River, and Caeté Rivers Regions. The highest mortality occurred in Região Metropolitana in 2011 ; and the lowest, in Região Guamá in the years 2009 and 2010. CONCLUSION: In the period studied, Pará State was far from reaching the indicators proposed for the elimination of TB, contributing to the maintenance of endemic in Brazil.
\end{abstract}

Keywords: Tuberculosis; Incidence; Program Evaluation; Ecological Studies.

\author{
Correspondência / Correspondence: \\ Dilma Costa de Oliveira Neves \\ Trav. Chaco, 2155. Bairro: Marco. CEP: 66093-572 - Belém, Pará, Brasil - Tel.: +55 (91) 99233-2525 \\ E-mail:dilmaconeves@gmail.com
}




\section{INTRODUÇÃO}

monitoramento e a avaliação, realizados pela coordenação do Programa Nacional de Controle da Tuberculose (PNCT), tomando por base as análises efetuadas pelos estados ou municípios, dificultam a identificação das fragilidades na condução do PNCT, em nível nacional, nos diferentes níveis da gestão em saúdele, Tais fragilidades podem ser decorrentes das desigualdades sociais que, intensificadas por fluxos migratórios e deficiências do próprio sistema de saúde, além do crescente número de casos de tuberculose (TB) multidrogas resistentes e a coinfecção pelo HIV, fortalecem a magnitude da TB como um problema de saúde pública ${ }^{3}$.

A incidência da TB em grupos populacionais que apresentam elevada vulnerabilidade social permite sua associação com a determinação social do processo saúde-doença ${ }^{4,5}$. A incorporação do PNCT pela atenção básica em saúde é considerada como alavanca para aprimorar o controle da endemia, visto que as ações desenvolvidas nesse nível de atenção à saúde são direcionadas aos grupos populacionais que possuem condicionantes para a disseminação da doença6. Por isso, no Pacto pela Vida, no Brasil, - controle da TB foi inserido como uma das seis prioridades a serem pactuadas entre os gestores do Sistema Único de Saúde (SUS), visando o impacto sobre a situação de saúde da população mediante o alcance de, pelo menos, $85 \%$ de cura de casos novos diagnosticados a cada ano ${ }^{7}$.

As metas propostas pelo PNCT são aferidas em indicadores epidemiológicos e operacionais a serem alcançados pelos serviços de saúde ${ }^{8}$. No entanto, esses indicadores não são adequados à realidade dos municípios considerados de pequeno porte e nem daqueles tidos como prioritários pelo PNCT, mas indicam a necessidade premente de uma abordagem mais qualificada e abrangente do problema pela rede de serviços da atenção básica9. Os indicadores epidemiológicos de impacto constantes no PNCT são os de morbidade e de mortalidade. As metas brasileiras, para 2035, são o alcance de menos de 10 casos novos de TB/100.000 hab. e de menos de um óbito por $\mathrm{TB} / 100.000$ hab. $^{10}$.

A avaliação e o monitoramento da efetividade das ações do PNCT são realizados mediante o alcance das metas pactuadas entre gestores municipais e estaduais de saúde ${ }^{11}$. São elas: a redução do abandono de tratamento para menos de 5\%; a detecção de 70\% dos casos pulmonares bacilíferos; e a cura de $85 \%$ dos casos notificados ${ }^{8}$.

Segundo o relatório da Organização Mundial da Saúde $(\mathrm{OMS})^{12}$ de 2016, o Brasil ocupava o $20^{\circ}$ lugar no ranking dos países com maiores incidências de TB. A estratégia proposta pela OMS para a eliminação da doença, juntamente com os objetivos do desenvolvimento sustentável, era a da eliminação da epidemia global em um período de 20 anos (2016-2035), com a redução da mortalidade e da morbidade a serem alcançadas em $2030^{13}$.
No estado do Pará, a alta por cura, entre o total de pacientes diagnosticados, encontrava-se abaixo dos 85\%; mas, se esse indicador estivesse associado ao abandono do tratamento, possibilitaria a crescente magnitude da TB no Estado 14,15.

Dos 16 municípios prioritários identificados na Região Norte, sete $(43,7 \%)$ encontravam-se no estado do Pará. São polos de desenvolvimento econômico e regional, o que possibilita, ao lado da geração de riqueza, um aumento das desigualdades sociais e da TB como problema sanitário a ser estudado, ao mesmo tempo requer acompanhamento dos indicadores de avaliação tanto epidemiológicos como operacionais do PNCT 10,14 .

Face à fragilidade apresentada pelo sistema de saúde no Brasil e no Pará, em decorrência das condições socioeconômicas favoráveis à transmissão e ao desenvolvimento da doença e daquelas desfavoráveis à condução das ações para seu controle, este estudo visou analisar alguns indicadores do PNCT no Pará, no período de 2005 a 2014.

\section{MATERIAIS E MÉTODOS}

Este estudo foi aprovado pelo Comitê de Ética em Pesquisa em Seres Humanos do Centro Universitário do Estado do Pará, sob o parecer de número 1.344.940, em $1^{\circ}$ de dezembro de 2015.

Trata-se de um estudo ecológico, com base em análise exploratória documental, utilizando dados de casos novos de TB pulmonar em pessoas com idade a partir de 20 anos, ocorridos no Pará, no período de 2005 a 2014, e registrados no Sistema de Informação de Agravos de Notificação (Sinan), sob a gerência da Secretaria de Saúde Pública do Estado do Pará (SESPA). Para o cálculo da incidência, foi utilizada a população censitária (2010) e as estimativas intercensitárias disponibilizadas pelo Instituto Brasileiro de Geografia e Estatística $^{16}$.

Para a construção dos indicadores epidemiológicos de avaliação do PNCT10,14, as variáveis utilizadas foram: Região de Integração de notificação; ano do diagnóstico da doença; situação de encerramento (cura, abandono e óbito); e realização da baciloscopia do escarro. Para os indicadores epidemiológicos de impacto, foram utilizados o número de casos novos notificados e o número de óbitos. E, para os indicadores epidemiológicos de resultado, a proporção de cura de casos novos com e sem baciloscopia positiva e a proporção de abandono de tratamento de casos novos com comprovação bacteriológica? .

○álculo da redução de incidência foi efetuado utilizando-se a subtração entre o valor inicial e o final, dividido pelo valor inicial, e o resultado multiplicado por 100. Para o aumento ou incremento da taxa de incidência ou de cura, o cálculo foi efetuado por meio da subtração entre o valor final e o inicial, dividido pelo valor inicial, e o resultado multiplicado por 100.

Foram incluídos os registros existentes no Sinan de casos novos de TB pulmonar, no período de 2005 a 
2014, com idade a partir de 20 anos e especificação do município de residência no Pará, além do preenchimento adequado de no mínimo 50\% das variáveis selecionadas para o estudo.

As regiões de notificação utilizadas foram as 12 Regiões de Integração do Pará, a saber: Araguaia, Baixo Amazonas, Carajás, Guamá, Lago de Tucuruí, Marajó, Metropolitana, Rio Caeté, Rio Capim, Tapajós, Tocantins e Xingu ${ }^{17}$.

Os dados foram ajustados por idade, tanto entre as regionais como intrarregionalmente, e analisados pela estatística descritiva, para a obtenção de média e desvio padrão. $\bigcirc$ teste Anova, para um critério com pós-teste de Tukey, foi utilizado para a comparação dos diferentes grupos regionais na série histórica de 10 anos. A análise estatística foi efetuada com auxílio do programa BioEstat $v 5.4^{18}$ e aceito, como diferença significativa, o valor de $p<0,05$ para $\alpha=5 \%$.

\section{RESULTADOS}

Foram notificados 40.990 casos, sendo que 40.882 $(99,7 \%)$ ocorreram em pessoas residentes no Pará, dos quais $36.358(88,9 \%)$ possuíam 20 anos ou mais de idade. Desses, 31.372 (86,3\%) foram casos de TB pulmonar que atenderam aos critérios de inclusão no estudo.

As Regiões de Integração Metropolitana e Guamá apresentaram, no período, as duas maiores incidências, 114,7 e 78,4 casos/10.000 hab. a partir de 20 anos de idade, respectivamente. Esses valores foram superiores à incidência global do Estado (71,7/10.000 hab.) na mesma faixa etária. Observa-se que a Região Metropolitana apresentou a maioria
$(49,6 \%)$ dos casos registrados e com o maior risco de adoecimento. Excetuando as Regiões Metropolitana e Guamá, nas demais Regiões a incidência, para o período, foi inferior a do Estado (Tabela 1).

Observa-se, ainda na tabela 1, que a Região Metropolitana apresentou valores de incidências médias que oscilaram entre 19,7 (2007-2008) e 25,8 (2013-2014) com tendência crescente, de 22,3\%, passando de 21,1 em 2005-2006 para 25,8 casos/10.000 pessoas a partir de 20 anos de idade em 2013-2014. Embora os valores das incidências médias nas demais Regiões e no Estado tenham oscilado ao longo do período, pode-se observar que houve uma redução no risco de adoecimento por TB pulmonar, ao se comparar a incidência média entre os anos de 2005-2006 e 2013-2014. As maiores reduções foram observadas na Região Xingu (41,9\%), seguida das Regiões Baixo Amazonas (35,8\%) e Araguaia (34,8\%). Não foi observada diferença estatística significativa ( $p=0,5656$ ) entre os valores de incidências médias entre os anos e entre as Regiões de Integração.

No período do estudo, dos 31.372 casos notificados, 22.216 pacientes receberam alta por cura, o que resulta em uma taxa de cura de 70,8\%. Essa taxa variou de 55,0\%, em 2014, na Região Tapajós, a 84,4\%, em 2005, na Região Rio Caeté. Na análise da comparação entre início e fim do período, foi observado incremento nas taxas de cura nas Regiões Araguaia (14,7\%), Carajás (20,3\%), Lago de Tucuruí $(12,3 \%)$ e Tocantins $(4,1 \%)$ (Tabela 2). Não foram observadas diferenças estatísticas significativas ( $p=0,7763$ ) entre os valores das taxas de cura entre as Regiões de Integração e os anos da série histórica estudada.

Tabela 1 - Distribuição da incidência média* de TB pulmonar em pacientes a partir 20 anos de idade, segundo as Regiões de Integração do estado do Pará, no período de 2005 a 2014

\begin{tabular}{|c|c|c|c|c|c|c|c|c|c|c|c|c|}
\hline Regiões & Casos & $\begin{array}{l}\text { Coef. } \\
\text { incid. } \\
\text { média } \\
2005- \\
2006\end{array}$ & Casos & $\begin{array}{l}\text { Coef. } \\
\text { incid. } \\
\text { média } \\
2007- \\
2008\end{array}$ & Casos & $\begin{array}{l}\text { Coef. } \\
\text { incid. } \\
\text { média } \\
2009- \\
2010\end{array}$ & Casos & $\begin{array}{l}\text { Coef. } \\
\text { incid. } \\
\text { média } \\
2011- \\
2012\end{array}$ & Casos & $\begin{array}{c}\text { Coef. } \\
\text { incid. } \\
\text { média } \\
2013- \\
2014\end{array}$ & $\begin{array}{c}\text { Total de } \\
\text { casos }\end{array}$ & $\begin{array}{l}\text { Coef. } \\
\text { incid. } \\
\text { média } \\
2005- \\
2014\end{array}$ \\
\hline Araguaia & 174 & 9,2 & 174 & 7,6 & 229 & 8,5 & 196 & 6,7 & 184 & 6,0 & 957 & 35,5 \\
\hline Baixo Amazonas & 453 & 14,8 & 374 & 10,6 & 262 & 7,1 & 347 & 9,2 & 363 & 9,5 & 1.799 & 49,0 \\
\hline Carajás & 301 & 12,8 & 311 & 11,0 & 307 & 9,7 & 311 & 9,1 & 365 & 10,1 & 1.595 & 50,4 \\
\hline Guamá & 525 & 18,3 & 514 & 15,4 & 530 & 15,0 & 554 & 15,1 & 647 & 17,3 & 2.770 & 78,4 \\
\hline Lago de Tucuruí & 189 & 11,7 & 185 & 9,9 & 194 & 9,9 & 145 & 7,0 & 181 & 8,4 & 894 & 45,7 \\
\hline Marajó & 169 & 4,0 & 215 & 4,8 & 224 & 9,6 & 215 & 4,3 & 201 & 3,9 & 1.024 & 43,7 \\
\hline Metropolitana & 2.543 & 21,1 & 2.647 & 19,7 & 3.252 & 24,0 & 3.506 & 25,5 & 3.624 & 25,8 & 15.572 & 114,7 \\
\hline Rio Caeté & 318 & 15,2 & 314 & 12,8 & 311 & 12,1 & 309 & 11,6 & 293 & 10,7 & 1.545 & 60,0 \\
\hline Rio Capim & 306 & 11,7 & 296 & 9,9 & 292 & 9,1 & 280 & 8,2 & 249 & 8,5 & 1.423 & 44,3 \\
\hline Tapajós & 178 & 14,8 & 190 & 13,5 & 153 & 11,4 & 155 & 12,1 & 159 & 11,5 & 835 & 62,1 \\
\hline Tocantins & 454 & 15,4 & 346 & 9,8 & 418 & 10,8 & 403 & 9,8 & 464 & 10,7 & 2.085 & 53,7 \\
\hline Xingu & 210 & 14,8 & 186 & 11,4 & 137 & 7,7 & 171 & 9,0 & 169 & 8,6 & 873 & 49,1 \\
\hline Estado & 5.820 & 15,2 & 5.752 & 13,2 & 6.309 & 14,4 & 6.592 & 13,8 & 6.899 & 14,1 & 31.372 & 71,7 \\
\hline
\end{tabular}


Tabela 2 - Distribuição anual das taxas (\%) de alta por cura dos casos de TB pulmonar notificados em pacientes a partir de 20 anos de idade, segundo as Regiões de Integração do estado do Pará, no período de 2005 a 2014

\begin{tabular}{|c|c|c|c|c|c|c|c|c|c|c|c|}
\hline \multirow{2}{*}{ Regiões } & \multicolumn{10}{|c|}{ Anos } & \multirow{2}{*}{ Média $\pm 2 d p$} \\
\hline & 2005 & 2006 & 2007 & 2008 & 2009 & 2010 & 2011 & 2012 & 2013 & 2014 & \\
\hline Araguaia & 62,3 & 70,3 & 75,9 & 55,4 & 66,4 & 65,1 & 69,7 & 58,0 & 69,3 & 71,4 & $66,4 \pm 6,3$ \\
\hline Baixo Amazonas & 75,7 & 83,2 & 82,0 & 72,2 & 76,1 & 71,4 & 77,0 & 75,7 & 76,0 & 67,7 & $75,7 \pm 4,6$ \\
\hline Carajás & 53,4 & 58,9 & 60,1 & 57,9 & 57,1 & 55,1 & 67,3 & 61,8 & 60,5 & 64,2 & $59,6 \pm 4,2$ \\
\hline Guamá & 79,3 & 80,5 & 77,7 & 78,1 & 78,0 & 71,4 & 81,7 & 74,4 & 80,4 & 77,6 & $77,9 \pm 3,1$ \\
\hline Lago de Tucuruí & 70,3 & 79,3 & 65,4 & 65,9 & 69,1 & 70,4 & 73,8 & 65,5 & 71,0 & 78,9 & $70,9 \pm 5,1$ \\
\hline Marajó & 74,7 & 76,7 & 75,7 & 80,7 & 70,2 & 77,4 & 76,0 & 77,9 & 67,8 & 69,5 & $74,6 \pm 4,2$ \\
\hline Metropolitana & 69,3 & 71,8 & 70,1 & 69,7 & 68,9 & 71,9 & 71,2 & 65,8 & 72,7 & 60,6 & $69,2 \pm 3,6$ \\
\hline Rio Caeté & 84,4 & 81,9 & 81,1 & 66,7 & 72,8 & 69,8 & 78,2 & 77,7 & 77,0 & 71,2 & $76,1 \pm 5,8$ \\
\hline Rio Capim & 76,2 & 70,3 & 75,6 & 76,3 & 76,6 & 65,3 & 71,1 & 75,7 & 75,7 & 63,8 & $72,7 \pm 4,8$ \\
\hline Tapajós & 70,1 & 79,8 & 63,0 & 67,0 & 68,6 & 72,9 & 60,5 & 70,0 & 58,7 & 55,0 & $66,6 \pm 7,4$ \\
\hline Tocantins & 74,4 & 76,3 & 78,4 & 79,6 & 78,8 & 76,1 & 77,0 & 76,3 & 80,6 & 77,4 & $77,5 \pm 0,6$ \\
\hline Xingu & 73,6 & 71,3 & 76,5 & 83,2 & 76,7 & 79,5 & 71,6 & 77,2 & 72,2 & 73,0 & $75,5 \pm 3,9$ \\
\hline Estado & 70,8 & 73,6 & 72,1 & 70,5 & 70,3 & 70,7 & 72,4 & 68,4 & 72,9 & 65,2 & $70,8 \pm 2,5$ \\
\hline
\end{tabular}

$p=0,7763$ (teste ANOVA)

Dentre os 31.372 casos novos de TB pulmonar incluídos no estudo, $22.774(72,6 \%)$ foram confirmados por baciloscopia. A taxa de cura entre os positivos foi de 73,3\% (16.690/22.774). A maior taxa foi observada na Região Rio Caeté $(85,4 \%)$, em 2006; e a menor taxa, na Região Tapajós (48,9\%), em 2014 (Tabela 3). Não foi constatada diferença estatística significativa $(p=0,4044)$ nas taxas entre as Regiões e entre os anos estudados.

Na análise comparativa das taxas de cura dos casos positivos, entre 2005 e 2014, observou-se um aumento nas Regiões Araguaia (17,9\%), Carajás (12,7\%), Lago de Tucuruí $(7,3 \%)$ e Tocantins (3,7\%).
As taxas de abandono de tratamento dos casos confirmados laboratorialmente variaram de 27,1\%, em 2008, na Região Carajás, a 2,5\%, em 2010, no Baixo Amazonas (Tabela 4). Observou-se um aumento das taxas de abandono em 66,7\% (8/12) das Regiões. Comparando-se os anos de 2005 e 2014, os maiores incrementos foram observados nas Regiões Xingu (230,9\%), Tapajós (196,0\%) e Marajó $(191,7 \%)$. Foram também observados aumentos nas taxas das Regiões Tocantins (13,0\%), Carajás (50,0\%), Rio Capim (5,3\%), Rio Caeté $(27,3 \%)$ e Guamá $(48,2 \%)$.

Tabela 3 - Evolução das taxas (\%) de alta por cura dos casos de TB pulmonar com baciloscopia positiva em pacientes com idade a partir de 20 anos nas Regiões de Integração do estado do Pará, no período de 2005 a 2014

\begin{tabular}{|c|c|c|c|c|c|c|c|c|c|c|c|}
\hline \multirow{2}{*}{ Regiões } & \multicolumn{10}{|c|}{ Anos } & \multirow{2}{*}{ Média $\pm 2 d p$} \\
\hline & 2005 & 2006 & 2007 & 2008 & 2009 & 2010 & 2011 & 2012 & 2013 & 2014 & \\
\hline Araguaia & 63,6 & 71,9 & 82,0 & 56,6 & 73,7 & 69,3 & 71,0 & 62,5 & 69,7 & 75,0 & $69,5 \pm 7,2$ \\
\hline Baixo Amazonas & 78,9 & 84,3 & 81,7 & 77,0 & 74,1 & 79,3 & 77,5 & 78,0 & 78,5 & 75,7 & $78,5 \pm 2,9$ \\
\hline Carajás & 65,9 & 80,6 & 80,7 & 70,1 & 73,9 & 71,9 & 83,7 & 75,6 & 71,9 & 74,3 & $74,9 \pm 5,5$ \\
\hline Guamá & 79,4 & 82,0 & 78,3 & 79,5 & 78,2 & 71,1 & 81,1 & 75,5 & 83,4 & 75,5 & $78,4 \pm 3,7$ \\
\hline Lago de Tucuruí & 73,9 & 80,3 & 69,3 & 59,7 & 68,5 & 72,6 & 76,9 & 74,2 & 71,6 & 79,3 & $72,6 \pm 6,0$ \\
\hline Marajó & 80,6 & 76,5 & 75,0 & 85,1 & 72,2 & 79,4 & 79,7 & 78,1 & 72,3 & 73,8 & $77,2 \pm 4,1$ \\
\hline Metropolitana & 69,7 & 73,3 & 70,9 & 72,3 & 70,5 & 73,5 & 73,6 & 67,4 & 72,8 & 61,9 & $70,6 \pm 3,6$ \\
\hline Rio Caeté & 85,1 & 85,4 & 80,4 & 63,5 & 72,2 & 73,2 & 77,5 & 77,4 & 79,2 & 75,8 & $76,9 \pm 6,5$ \\
\hline Rio Capim & 78,9 & 71,6 & 80,6 & 74,8 & 76,8 & 72,0 & 75,0 & 77,5 & 79,0 & 69,4 & $75,5 \pm 3,7$ \\
\hline Tapajós & 70,1 & 81,0 & 67,7 & 80,7 & 71,2 & 79,6 & 64,0 & 72,9 & 57,4 & 48,9 & $69,4 \pm 10,4$ \\
\hline Tocantins & 75,0 & 77,5 & 76,4 & 81,0 & 78,4 & 79,1 & 77,2 & 79,1 & 80,5 & 77,8 & $78,2 \pm 1,8$ \\
\hline Xingu & 76,4 & 79,7 & 76,7 & 82,0 & 81,8 & 82,5 & 75,4 & 81,8 & 72,4 & 76,4 & $78,5 \pm 3,5$ \\
\hline Estado & 73,1 & 76,5 & 74,4 & 73,5 & 72,7 & 74,1 & 75,3 & 71,1 & 74,5 & 67,3 & $73,3 \pm 2,6$ \\
\hline
\end{tabular}


Tabela 4 - Evolução das taxas (\%) de abandono de tratamento nos casos de TB pulmonar com baciloscopia positiva em pacientes com idade a partir de 20 anos nas Regiões de Integração do estado do Pará, no período de 2005 a 2014

\begin{tabular}{|c|c|c|c|c|c|c|c|c|c|c|c|}
\hline \multirow{2}{*}{ Regiões } & \multicolumn{10}{|c|}{ Anos } & \multirow{2}{*}{ Média $\pm 2 d p$} \\
\hline & 2005 & 2006 & 2007 & $2008^{*}$ & 2009 & 2010 & 2011 & 2012 & 2013 & 2014 & \\
\hline Araguaia & 19,7 & 10,9 & 6,6 & 22,6 & 14,5 & 12,0 & 6,5 & 12,5 & 10,6 & 15,6 & $13,2 \pm 5,2$ \\
\hline Baixo Amazonas & 9,9 & 8,2 & 5,5 & 8,9 & 4,6 & 2,5 & 5,9 & 5,5 & 4,4 & 5,2 & $6,1 \pm 2,3$ \\
\hline Carajás & 11,6 & 16,1 & 12,3 & 27,1 & 22,8 & 16,7 & 6,2 & 18,7 & 18,8 & 17,4 & $16,8 \pm 5,9$ \\
\hline Guamá & 8,3 & 6,9 & 11,3 & 9,8 & 11,4 & 10,4 & 10,6 & 11,0 & 10,8 & 12,3 & $10,3 \pm 1,6$ \\
\hline Lago de Tucuruí & 11,6 & 13,6 & 20,0 & 22,6 & 12,3 & 13,7 & 11,5 & 9,7 & 14,9 & 3,4 & $13,3 \pm 5,3$ \\
\hline Marajó & 6,0 & 16,2 & 9,5 & 14,9 & 15,3 & 10,3 & 13,9 & 12,5 & 18,5 & 17,5 & $13,5 \pm 3,9$ \\
\hline Metropolitana & 14,4 & 14,6 & 17,6 & 16,6 & 16,1 & 12,6 & 11,6 & 12,7 & 13,6 & 12,8 & $14,3 \pm 2,0$ \\
\hline Rio Caeté & 6,6 & 5,8 & 12,8 & 18,3 & 15,8 & 9,4 & 7,5 & 11,3 & 11,7 & 8,4 & $10,8 \pm 4,1$ \\
\hline Rio Capim & 13,2 & 18,9 & 7,4 & 13,0 & 11,6 & 10,8 & 8,0 & 6,3 & 11,1 & 13,9 & $11,7 \pm 3,8$ \\
\hline Tapajós & 7,5 & 7,9 & 10,8 & 10,5 & 16,9 & 8,2 & 10,0 & 10,0 & 21,3 & 22,2 & $12,5 \pm 5,5$ \\
\hline Tocantins & 9,2 & 17,5 & 12,7 & 13,9 & 11,1 & 17,3 & 10,1 & 13,4 & 14,8 & 10,4 & $13,0 \pm 2,9$ \\
\hline Xingu & 5,5 & 14,5 & 15,1 & 11,5 & 7,3 & 9,5 & 10,8 & 10,9 & 13,8 & 18,2 & $11,4 \pm 3,7$ \\
\hline Estado & 12,0 & 13,3 & 14,4 & 15,7 & 14,6 & 11,9 & 10,5 & 12,0 & 13,3 & 12,6 & $13,0 \pm 1,6$ \\
\hline
\end{tabular}

$p=0,0325$ (teste ANOVA - Tukey).

Em todo o período estudado, a taxa de abandono foi de $13,9 \%(3.176 / 22.774)$. Foi observada diferença estatística significativa $(p=0,0325)$ nas taxas entre as Regiões e entre os anos estudados. $O$ ano de 2008 foi o que apresentou diferença significativa estatisticamente em relação aos demais anos estudados.

No período estudado, foram registrados 770 óbitos por TB pulmonar. A maior mortalidade foi observada na Região Metropolitana, com variação de 10,9 óbitos/100.000 hab. com idade a partir de 20 anos em 2006 para 29,6 em 2011. A menor taxa de mortalidade foi 0,1/100.000 hab. em 2009 e 2010, na Região Guamá. Em 2005, não foram encontrados registros de óbitos em nove das 12 Regiões (Tabela 5).

\section{DISCUSSÃO}

A TB ainda se apresenta como um dos maiores problemas de saúde pública no Brasil. A incidência tende a diminuir ${ }^{11,15}$; no entanto, a mortalidade ainda é elevada, principalmente na coinfecção com o vírus da imunodeficiência humana (HIV) ${ }^{19}$ e naqueles casos em que a vulnerabilidade social se faz presente ${ }^{11,15,19,20}$.

Tabela 5 - Evolução da mortalidade* por TB pulmonar em pessoas a partir de 20 anos de idade nas Regiões de Integração do estado do Pará, no período de 2005 a 2014

\begin{tabular}{|c|c|c|c|c|c|c|c|c|c|c|}
\hline \multirow{2}{*}{ Regiões } & \multicolumn{10}{|c|}{ Anos } \\
\hline & 2005 & 2006 & 2007 & 2008 & 2009 & 2010 & 2011 & 2012 & 2013 & 2014 \\
\hline Araguaia & 0,5 & 1,1 & 0,5 & 0,8 & 1,2 & 1,8 & 1,4 & 2,4 & 0,3 & 1,0 \\
\hline Baixo Amazonas & 0,7 & 1,0 & 3,1 & 2,0 & 0,8 & 1,1 & 0,8 & 0,8 & 1,0 & 0,8 \\
\hline Carajás & - & 0,4 & 0,7 & 0,7 & 1,6 & 3,0 & 1,5 & 1,7 & 2,5 & 1,4 \\
\hline Guamá & - & - & 0,4 & 0,5 & 0,1 & 0,1 & - & 0,4 & 0,2 & 0,3 \\
\hline Lago de Tucuruí & - & 0,3 & 0,3 & 1,5 & 0,9 & 0,6 & 1,1 & 1,1 & 0,8 & - \\
\hline Marajó & - & 0,5 & 2,1 & 0,4 & 0,8 & 0,8 & 1,9 & 0,4 & 1,1 & 0,7 \\
\hline Metropolitana & - & 10,9 & 27,4 & 27,0 & 29,2 & 23,1 & 29,6 & 24,0 & 20,9 & 14,2 \\
\hline Rio Caeté & - & 0,5 & 0,5 & 1,1 & 1,3 & 3,8 & 1,4 & 0,8 & 0,2 & 0,6 \\
\hline Rio Capim & - & - & 1,0 & 1,3 & 0,3 & 1,8 & 0,9 & 1,4 & 0,8 & 0,9 \\
\hline Tapajós & - & 0,8 & 3,7 & 1,4 & 3,3 & 3,4 & 5,0 & 4,4 & 3,6 & 1,4 \\
\hline Tocantins & - & 0,7 & 2,0 & 3,0 & 1,6 & 1,0 & 0,7 & 0,7 & 2,6 & 1,6 \\
\hline Xingu & 0,7 & - & 1,2 & 0,6 & 0,6 & 2,7 & 0,5 & 1,0 & 2,6 & - \\
\hline Estado & 0,1 & 0,8 & 2,2 & 2,2 & 2,1 & 2,2 & 2,1 & 2,0 & 1,9 & 1,3 \\
\hline
\end{tabular}


Embora com valores oscilando tanto intra como inter-regiões, os achados das taxas de incidência nas Regiões de Integração do Pará, como um indicador epidemiológico de impacto do $\mathrm{PNCT}^{8,11}$, demonstraram tendência à redução; à exceção da Região Metropolitana, pois, das elevadas taxas de incidência, também apresentou tendência de crescimento. Um estudo comparativo, para o período de 2005 a 2014, das taxas de incidência entre as regiões metropolitanas e a média nacional brasileira detectou que nove metrópoles, entre elas Belém (83,2/100.000 hab.), apresentaram taxas superiores à média nacional, de $33,54 / 100.000$ hab. no ano de $2014^{21}$.

Nesse contexto, as taxas de incidência de TB, encontradas na Região Metropolitana, podem estar relacionadas à presença crescente de áreas populacionais carentes nos cinco municípios que a compõem, incluindo a cidade de Belém, o que favorece o aumento da incidência ${ }^{22}$. Tais achados podem ser decorrentes da própria dinâmica da vida na cidade, que por um lado pode apresentar maior facilidade de acesso aos programas e serviços de saúde, mas por outro detém os desafios da elevada densidade demográfica, das alterações ambientais e das relações sociais que se apresentam como fatores de risco à saúde das pessoas ${ }^{20}$.

Embora tenha sido observada maior redução da incidência, em algumas Regiões do Estado, resultado provavelmente de iniciativas adequadas no combate e prevenção da doença, o Pará encontra-se entre os nove estados brasileiros com incidência de casos de TB superior à média nacional de 32,4/100.000 hab. ${ }^{10}$. A redução dos casos novos de TB observada foi semelhante à média nacional, de acordo com a análise de tendência do período de 2003 a $2012^{14}$, em que o coeficiente de incidência da doença mostrou declínio de $2 \%$ ao ano. Mesmo com esse declínio, o Brasil encontra-se entre os 20 países com elevada carga da doença, conforme o estimado pela OMS para o período 2016-202012.

A redução da incidência, observada nas Regiões de Integração, também pode ser em decorrência do aumento da cobertura da Estratégia Saúde da Família (ESF) e da descentralização do tratamento diretamente observado", visto que houve incorporação do monitoramento da TB nas ações da atenção básica. Porém, tal expansão necessita estar acompanhada da redução das fragilidades apresentadas pela ESF como porta de entrada no sistema de saúde, tais como no acesso, acolhimento e estabelecimento/manutenção do vínculo usuário-serviço. Essas fragilidades interferem na busca por esses serviços pelos pacientes que necessitam de atenção ao seu problema de saúde ${ }^{23}$.

Apesar de ter ocorrido aproximadamente $130 \%$ de expansão da cobertura da ESF no Pará, de 21,3\% em 2004 para 48,9\% em 2014, a mesma ainda estava abaixo da meta do Ministério da Saúde (MS) e se distribuiu de forma heterogênea entre os municípios e regionais ${ }^{24}$. Esse fato pode comprometer o achado da tendência de redução da incidência na maioria das Regiões de Integração com base nos registros do Sinan, o que pode ser motivo de preocupação para os coordenadores do PNCT, no nível de gestão municipal e estadual, uma vez que dados ausentes ou inconsistentes prejudicam a análise anual dos indicadores de impacto do Programa. Tal fato requer que esses coordenadores realizem anualmente a avaliação e o monitoramento in loco, a fim de identificar possíveis entraves na operacionalização do Programa, utilizando os indicadores operacionais de processo e de resultado ${ }^{11}$.

Outro fator a ser considerado, na implementação e na análise dos indicadores de impacto das ações do PNCT, é a relação entre a ocorrência da TB e a vulnerabilidade social. Nessa análise devem ser inseridos os baixos Índices de Desenvolvimento Humano Municipal, predominantes na maioria dos municípios paraenses $^{25}$, o que potencialmente dificulta o entendimento do paciente tanto sobre a doença quanto sobre o seu tratamento, além de contribuir para o não alcance da meta estabelecida pelo MS para o controle da doença ${ }^{14}$.

Dessa forma, para que os países alcancem o fim da epidemia até 2035, faz-se necessário ter como metas a redução da incidência em $90 \%$ e da mortalidade em 95\%, sendo que essas reduções deverão tomar por base o valor desses indicadores no ano de $2015^{9}$. Essas metas devem ser progressivamente alcançadas, assegurando que as famílias recebam o tratamento gratuito, sem onerar seus ganhos com custos referentes ao tratamento específico para TB, e minimização dos efeitos colaterais do mesmo. É considerado que os gastos com tratamento contribuem para um maior empobrecimento dos doentes, o que se torna um empecilho para o acesso aos serviços de saúde, acarretando ausências de tratamento e do adequado acompanhamento?.

O MS classifica a taxa de alta por cura como um indicador epidemiológico de resultado, por permitir avaliações em intervalos de tempo menores que os indicadores de impacto. Para tanto, foi estabelecida, como parâmetro nacional, uma taxa igual ou superior a $85 \%$ a ser utilizada na pactuação entre os gestores municipais e estaduais, visando à redução da transmissibilidade do bacilo de Koch e, em consequência, a incidência ${ }^{1,11,26}$.

Ainda que a elevação da taxa de cura (com e sem confirmação bacteriológica) tenha ocorrido em 50\% das 12 Regiões de Integração, quando comparados os valores de 2005 e 2014, observa-se que nenhuma delas alcançou a meta pactuada ao final do período estudado. Em 2014, o Pará atingiu 65,2\% de alta por cura, portanto 19,8 pontos percentuais abaixo da meta de $85 \%$ estabelecida pelo MS. Esse achado foi semelhante ao estudo de coorte de 311 pacientes, realizado na cidade do Rio de Janeiro, no período de 2004 a 2006, no qual a taxa de cura encontrada foi de $72 \%{ }^{27}$. Tais estudos reforçaram que a redução da epidemia ainda se encontrava muito aquém das metas estabelecidas no pacto da atenção básica. 
Apenas as Regiões Rio Caeté, em 2005, e Marajó, em 2008, apresentaram taxas de cura em pacientes com baciloscopia positiva compatível com o parâmetro estabelecido pelo MS. De um modo geral, as baixas taxas de cura, em casos com confirmação bacteriológica, podem ter sido resultado da fragilidade dos municípios quanto ao registro das baciloscopias realizadas nas unidades básicas de saúde ou nos serviços de média complexidade. Segundo - MS, compete ao estado realizar a supervisão e o monitoramento dos exames laboratoriais realizados na rede do SUS, a fim de assegurar a qualidade dos mesmos. Compete também ao estado suprir a fragilidade dos municípios quanto à realização de baciloscopia, cultura, identificação do bacilo e teste de sensibilidade às drogas utilizadas no tratamento da $T B$, visando ao fortalecimento da execução das ações e ao alcance das metas pactuadas entre os gestores do SUS ${ }^{8}$.

O alcance da taxa de cura é a principal estratégia para a redução da morbimortalidade da $\mathrm{TB}^{26}$, principalmente entre os pacientes bacilíferos ${ }^{8,11}$. Sua avaliação reflete a qualidade dos serviços prestados aos pacientes, assim como serve de apoio à tomada de decisão pelos gestores em saúde. Por isso, ante a suspeita clínica, torna-se indispensável submeter os pacientes a diagnóstico e tratamento corretos, a fim de que seja alcançada a meta de redução da incidência proposta pelo $\mathrm{MS}^{28,29}$.

Dessa forma, as baixas taxas de cura somadas à elevada taxa de abandono em pacientes com baciloscopia positiva refletem a necessidade, no estado, de aumento da qualidade na cobertura do tratamento diretamente observado associado a maior cobertura populacional pela ESF9. $O$ abandono de tratamento, na maioria das Regiões (10), esteve acima de 5\%, ou seja, superior ao mínimo aceitável pela $\mathrm{OMS}^{12,13}$. A melhoria da qualidade das ações do PNCT possui como desafios o estigma, o preconceito, a incapacidade do doente em continuar o tratamento, a associação com a pobreza e a impossibilidade da cura ou a permanência de sequelas como representações sociais inerentes ao paciente e à doença ${ }^{3}$.

Embora a proporção de abandono seja um dos principais fatores que interferem na taxa de cura, outros devem ser considerados para o não alcance desse desfecho favorável ao tratamento, como a coinfecção TB-HIV, o alcoolismo, a falência de tratamento ${ }^{27}$, o aumento das taxas de recidiva e mortalidade, além do desenvolvimento de multirresistência aos fármacos ${ }^{28}$. Associa-se a esses fatores a fragilidade da estrutura dos serviços de saúde para ampliação tanto da busca ativa dos sintomáticos respiratórios como dos exames de contatos dos casos índice e confirmação laboratorial $^{20,28}$.

Para que ocorram a redução das taxas de abandono e o aumento das taxas de cura, são necessárias a organização e a adequação da rede de serviços para o cumprimento das competências municipais quanto à operacionalização do PNCT. A coordenação municipal do Programa deve empregar esforços no sentido de efetivamente realizar atividades de prevenção, busca de faltosos, avaliação dos contatos, interação do Programa com outros setores afins e assegurar a disponibilidade dos medicamentos, além de implementar estratégias para a adequada mobilização da sociedade na identificação das fragilidades e das fortalezas existentes na execução do Programa ${ }^{7}$. Nesse contexto, inserem-se a ESF e o agente comunitário de saúde (ACS), pois, em toda visita domiciliar e reuniões com a comunidade, - ACS, adequadamente treinado, poderá identificar sintomáticos respiratórios e encaminhá-los para a unidade de saúdé .

Para o MS, o elevado número de óbitos e de abandonos é consequência do difícil manejo clínico do paciente com $\mathrm{TB}^{28}$, o que reflete, em segmentos vulneráveis da população, a incidência do problema, associada à baixa efetividade do Programa ${ }^{11}$. Por isso, a redução da taxa de mortalidade consiste em um desses desafios, mesmo que se tenha observado, no Brasil, redução do coeficiente de mortalidade por TB no período de 2004 a 2014. Nesse período, as capitais brasileiras com maior risco de morte por TB foram: Recife (Pernambuco), Rio de Janeiro (Rio de Janeiro) e Belém (Pará) ${ }^{15}$.

Pode-se dizer que a piora do quadro clínico, podendo levar ao óbito, é decorrente da baixa escolaridade, da baixa renda e de outros fatores socioeconômicos que, quando associados, levam ao pouco conhecimento da doença, o que, em um contexto de desigualdade de acesso, retarda a busca pelo serviço de saúde ${ }^{30}$. Nesse sentido, destacam-se hábitos de vida, como o consumo de álcool, drogas ilícitas e fumo, reduzindo ou atrasando a busca aos serviços de saúde pelos pacientes, a necessidade do adequado conhecimento sobre a doença e seu tratamento, a fim de reduzir o estigma e o impacto social da TB ${ }^{31}$. Além disso, vale ressaltar a deficiência nutricional desses pacientes, o que denota a necessidades e a importância do acompanhamento nutricional durante o tratamento da $\mathrm{TB}^{32}$.

Embora se descrevesse um cenário que pudesse ser favorável ao aumento da mortalidade, foram encontradas, em 2014, em 91,7\% (11) das Regiões, taxas de mortalidade inferiores a 2,5/100.000 hab. (parâmetro mínimo definido como um dos critérios para a seleção de município prioritário para - controle da $\mathrm{TB})^{14}$. Esses resultados diferiam da mortalidade por TB pulmonar encontrada nos registros do Sistema de Informação de Mortalidade (SIM). Para as Regiões Metropolitana, Rio Capim, Rio Caeté e Tocantins, houve aumento da mortalidade. Para - Estado, esse aumento foi de $21,2 \%$ de 2005 (3,3/100.000 hab.) para 2014 (4,4/100.000 hab.) e, em todos os anos estudados, a taxa foi superior a esse parâmetro ${ }^{33}$.

No Pará, em dezembro de 2015, a cobertura da ESF consistia em 43,2\%, sendo a maior cobertura registrada na Região Tocantins $(69,1 \%)$ e a menor na Região Xingu $(19,9 \%)^{34}$. Essas coberturas são inferiores 
ao necessário para o estabelecimento de impacto na saúde da comunidade. Ademais, a falta de atualização dos profissionais quanto ao diagnóstico, tratamento e acompanhamento dos casos novos, novas estratégias para vigilância dos contatos e busca de sintomáticos respiratórios, além da sobrecarga de trabalho decorrente de outras demandas da comunidade impedem o desenvolvimento de ações voltadas à própria comunidade, como as de educação em saúde 29,35,36.

Segundo o $\mathrm{MS}^{10}$, os indicadores epidemiológicos de impacto e de resultados, necessários ao monitoramento e à avaliação das ações de controle da endemia da TB, são extraídos de dados constantes no Sinan. No entanto, é necessário que a análise da qualidade desses dados seja sistematicamente realizada, visando identificar as falhas neles existentes, principalmente aquelas com registro ignorado ou não preenchido, o que foi excluído neste estudo.

Para o enfrentamento e controle da TB, o PNCT possui desafios, uma vez que depende de serviços de saúde com recursos de apoio diagnóstico e terapêutico adequados, recursos humanos qualificados e dedicados, integrantes de equipes multidisciplinares, que desenvolvam suas atividades diretamente nas comunidades e que sejam apoiados por sistemas de informações adequados ${ }^{36}$.

As limitações próprias do desenho metodológico deste estudo podem ter sido decorrentes da completude insatisfatória no preenchimento da ficha de notificação, assim como da ficha de acompanhamento e evolução do paciente, indicando a necessidade de um maior investimento do Estado para capacitação dos responsáveis pela notificação, assim como dos coordenadores municipais do Programa para compatibilização das informações de óbito por TB no Sinan e no SIM. Mesmo com a existência de tais limitações, identificaram-se contribuições para o PNCT nas Regiões de Integração do Pará, uma vez que, além de apontar as deficiências dos indicadores operacionais na avaliação e no monitoramento do Programa, reforçaram a fragilidade da organização da atenção básica no seu desenvolvimento.

\section{CONCLUSÃO}

Mesmo com a redução, no período deste estudo, da incidência média no Pará e em 83,3\% das Regiões de Integração, os indicadores analisados não foram alcançados, podendo refletir uma baixa prioridade para o controle da TB no sistema de saúde. A baixa taxa de cura, a elevada taxa de abandono entre os pacientes com baciloscopia positiva e a mortalidade registradas denotam que o Estado ainda contribuía para a endemia no país, assim como se encontrava distante do alcance dos valores dos indicadores estabelecidos como aceitáveis para o controle dessa doença.

\section{CONFLITOS DE INTERESSE}

Os autores declaram que não houve conflito de interesses.

\section{CONTRIBUIÇÃO DOS AUTORES}

Todos os autores contribuíram com a idealização do estudo, a análise e a interpretação dos dados e com a redação do manuscrito, aprovando a versão final publicada. Declaram-se responsáveis pelo conteúdo integral do artigo, garantindo sua precisão e integridade.

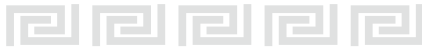

\section{REFERÊNCIAS}

1 Ministério da Saúde (BR). Secretaria de Vigilância em Saúde. Departamento de Vigilância das Doenças Transmissíveis. Avaliação da gestão do programa nacional de controle da tuberculose. Brasília: Ministério da Saúde; 2015. 36 p.

2 Heufemann NEC, Gonçalves MJF, Garnelo ML. Avaliação do programa de controle da tuberculose em cenário amazônico: desafios em Coari. Acta Amaz. 2013 mar;43(1):33-42.

3 Barreira D, Grangeiro A. Avaliação das estratégias de controle da tuberculose no Brasil. Rev Saude Publica. 2007 set;41 supl 1:4-8.

4 Ministério da Saúde (BR). Secretaria de Ciência, Tecnologia e Insumos Estratégicos. Departamento de Ciência e Tecnologia. Doenças negligenciadas: estratégias do Ministério da Saúde. Rev Saude Publica. 2010 fev;44(1): 200-2.
5 Bertolozzi MR, Takahashi RF, Hino P, Litvoc M, França FOS. $O$ controle da tuberculose: um desafio para a saúde pública. Rev Med (Sao Paulo). 2014 abr-jun;93(2):83-9.

6 Ministério da Saúde (BR). Secretaria de Vigilância em Saúde. Departamento de Vigilância das Doenças Transmissíveis. Cartilha para o agente comunitário de saúde: tuberculose. Brasília: Ministério da Saúde; 2017. 40 p.

7 Ministério da Saúde (BR). Secretaria Executiva. Departamento de Apoio à Descentralização. Diretrizes operacionais: pactos pela vida, em defesa do SUS e de gestão. Brasília: Ministério da Saúde; 2006. 76 p. (Série A. Normas e manuais técnicos).

8 Ministério da Saúde (BR). Secretaria de Vigilância em Saúde. Departamento de Vigilância Epidemiológica. Programa Nacional de Controle da Tuberculose [Internet]. Brasília: Ministério da Saúde; 2004 [citado 2017 jan 13]. Disponível em: http://bvsms.saude.gov. br/bvs/publicacoes/ProgramaTB.pdf. 
9 Ministério da Saúde (BR). Secretaria de Vigilância em Saúde. Departamento de Vigilância das Doenças Transmissíveis. Brasil livre da tuberculose: Plano Nacional pelo Fim da Tuberculose como Problema de Saúde Pública. Brasília: Ministério da Saúde; 2017.52 p.

10 Ministério da Saúde (BR). Secretaria de Vigilância em Saúde. Indicadores prioritários para $\mathrm{O}$ monitoramento do Plano Nacional pelo Fim da Tuberculose como Problema de Saúde Pública no Brasil. Bol Epidemiol. 2017;48(8):1-11.

11 Ministério da Saúde (BR). Secretaria de Vigilância em Saúde. Departamento de Vigilância Epidemiológica. Manual de recomendações para o controle da tuberculose no Brasil. Brasília: Ministério da Saúde; 2011. 284 p. (Série A. Normas e manuais técnicos).

12 World Health Organization. Global tuberculosis report 2016 [Internet]. Geneva: WHO; 2016 [cited 2017 May 10]. Available from: http://apps.who.int/ medicinedocs/en/d/Js23098en/.

13 World Health Organization. Bending the curve - ending TB: annual report 2017 [Internet]. New Delhi: WHO; 2017 [cited 2018 Mar 10]. Available from: http://apps.who.int/iris/ handle/10665/254762.

14 Ministério da Saúde (BR). Secretaria de Vigilância em Saúde. Departamento de Vigilância das Doenças Transmissíveis. Panorama da tuberculose no Brasil: indicadores epidemiológicos e operacionais. Brasília: Ministério da Saúde; 2014. $92 \mathrm{p}$.

15 Ministério da Saúde (BR). Secretaria de Vigilância em Saúde. Departamento de Vigilância das Doenças Transmissíveis. Panorama da tuberculose no Brasil: a mortalidade em números. Brasília: Ministério da Saúde; 2016. 126 p. Disponível em: http://portalarquivos2.saude.gov.br/images/ pdf/2016/outubro/15/panorama_tuberculose_ brasil_mortalidade.pdf.

16 Instituto Brasileiro de Geografia e Estatística. Censo demográfico 2010: aglomerados subnormais [Internet]. Rio de Janeiro: IBGE; 2010 [citado 2018 nov 2]. Disponível em: https://ww2.ibge. gov.br/home/estatistica/populacao/censo2010/ aglomerados_subnormais/default_aglomerados subnormais.shtm.

17 Ministério Público Federal (BR). Procuradoria da República no Pará. Decreto Estadual n. 1.066, de 19 de junho de 2008 [Internet]. Belém: MPF; 2016 [citado 2016 mar 20]. Disponível em: http://www. prpa.mpf.mp.br/setorial/biblioteca/legislacao/decr eto-estadual-n-1-066-de-19-de-junho-de-2008.

18 Ayres M, Ayres Jr M, Ayres DL, Santos AS. BioEstat 5.4: aplicações estatísticas nas áreas das ciências biológicas e médicas. Belém: UFPA; 2014. (Software).
19 Ministério da Saúde (BR). Secretaria de Vigilância em Saúde. Tuberculose no Brasil: realidade e perspectivas. Bol Epidemiol. 2012;43(1):16-9.

20 Basta PC, Marques M, Oliveira RL, Cunha EAT, Resendes APC, Souza-Santos R. Desigualdades sociais e tuberculose: análise segundo raça/cor, Mato Grosso do Sul. Rev Saude Publica. 2013 out;47(5):854-64.

21 Segurado AC, Cassenote AJ, Luna EA. Saúde nas metrópoles: doenças infecciosas. Estud Av. 2016 jan-mar;30(86):29-49.

22 Lima SS, Vallinoto ACR, Machado LFA, Ishak MOG, Ishak R. Análise espacial da tuberculose em Belém, estado do Pará, Brasil. Rev Pan-Amaz Saude. 2017 jun;8(2):57-65.

23 Mizuhira VF, Gazetta CE, Vendramin SHF, Ponce MAZ, Wysocki AD, Villa TCS. Procura da atenção básica para o diagnóstico da tuberculose. Arq Cienc Saude. 2015 abr-jun;22(2):94-8.

24 Ministério da Saúde (BR). Departamento de Atenção Básica. Proporção de cobertura populacional estimada por equipes de saúde da família [Internet]. 2018 [citado 2018 jun 14]. Disponível em: http://dab2. saude.gov.br/sistemas/notatecnica/frmListaMunic.php.

25 Programa das Nações Unidas para o Desenvolvimento. Instituto de Pesquisa Econômica Aplicada. Fundação João Pinheiro. Atlas do desenvolvimento humano no Brasil: índice de desenvolvimento humano municipal brasileiro [Internet]. 2010 [citado 2018 jun 12]. Disponível em: http://atlasbrasil.org.br/2013/pt/ranking.

26 Ministério da Saúde (BR). Caderno de diretrizes, objetivos, metas e indicadores. Brasília: Ministério da Saúde; 2016. 73 p.

27 Orofino RL, Brasil PEA, Trajman A, Schmaltz CAS, Dalcomo M, Rolla VC. Preditores dos desfechos do tratamento da tuberculose. J Bras Pneumol. 2012 jan-fev;38(1):88-97.

28 Ministério da Saúde (BR). Secretaria de Vigilância em Saúde. Detectar, tratar e curar: desafios e estratégias brasileiras frente à tuberculose. Bol Epidemiol. 2015;46(9):1-19.

29 Maciel ELN, Sales CMM. A vigilância epidemiológica da tuberculose no Brasil: como é possível avançar mais? Epidemiol Serv Saude. 2016 jan-mar;25(1):175-8.

30 Yamamura M, Santos-Neto M, Santos RAN, Garcia MCC, Nogueira JA, Arcêncio RA. Epidemiological characteristics of cases of death from tuberculosis and vulnerable territories. Rev Latino-Am Enfermagem. 2015 Sep-Oct;23(5):910-8.

31 Silva CCAV, Andrade MS, Cardoso MD. Fatores associados ao abandono do tratamento de tuberculose em indivíduos acompanhados em unidades de saúde de referência na cidade do Recife, Estado de Pernambuco, Brasil, entre 2005 e 2010. Epidemiol Serv Saude. 2013 mar;22(1):77-85. 
32 Piva SGN, Costa MCN, Barreto FR, Pereira SM. Prevalência de deficiência nutricional em pacientes com tuberculose pulmonar. J Bras Pneumol. 2013 jul-ago;39(4):476-83.

33 Ministério da Saúde (BR). Departamento de Informática do Sistema Único de Saúde. TabNet Win32 3.0: Mortalidade - Pará [Internet]. Brasília: Datasus; 2017 [citado 2018 jun 18]. Disponível em: http://tabnet. datasus.gov.br/cgi/deftohtm.exe?sim/cnv/obt10pa.def.

34 Ministério da Saúde (BR). Departamento de Informática do Sistema Único de Saúde. Informações de Saúde (TABNET): Assistência à Saúde - Atenção Básica Saúde da Família - de 1998 a 2015 [Internet]. Brasília: Datasus; 2016 [citado 2017 mar 1]. Disponível em: http://www2.datasus.gov.br/DATASUS/index. php? area $=0202 \& i d=11641 \& \mathrm{VObj}=\mathrm{http}: / / \mathrm{tabnet}$. datasus.gov.br/cgi/deftohtm.exe?siab/cnv/SIABF.
35 Salzani MGB, Oliveira SAC, Rocha MAZP, Jesus GJ, Gazetta CE, Vendramini SHF, et al. Diagnóstico de tuberculose: perspectiva do profissional de enfermagem da atenção primária. Refacs. 2017;5(2): 180-90.

36 Chiaverini DH, organizadora. Guia prático de matriciamento em saúde mental [Internet]. Brasília: Ministério da Saúde; 2011 [citado 2018 fev 19]. Disponível em: http://bvsms.saude.gov. br/bvs/publicacoes/guia_pratico_matriciamento saudemental.pdf. 